\title{
Uncertainties in TIR Hyperspectral Image Cube Unmixing
}

\author{
Keshav Dev Singh ${ }^{1,2}$ \\ ${ }^{1}$ Department of Earth Sciences, Indian Institute of Technology Bombay, Powai, Mumbai-400076, India \\ ${ }^{2}$ College of Agricultural and Environmental Sciences, University of California, Davis, CA 95616, USA
}

Copyright (C) 2017 by authors, all rights reserved. Authors agree that this article remains permanently open access under the terms of the Creative Commons Attribution License 4.0 International License

\begin{abstract}
The spectral unmixing in the thermal infrared (TIR) region is not a linear case. For simplicity, the spectral mixing for pixel deconvolution is assumed to be linear, but in reality, the intimate mixture spectra are non-linear. The multiple scattering effects due to texture and fabric do affect the spectral shape and form. In this paper, the uncertainties in unmixing of TIR hyperspectral image cube are investigated.
\end{abstract}

Keywords Thermal Infrared, Hypercube, Non-linearity, Spectral Unmixing

\section{Introduction}

Hyperspectral remote sensing in the thermal infrared region $(2-14 \mu \mathrm{m})$ is a very recent development. Rocks and minerals typically provide diagnostic spectral features only in the thermal region. The spectral range of 2.5 to $13.5 \mu \mathrm{m}$ is specifically dominated by emitted radiations and perfectly suited for spectral features detection [1]. This study addresses the problem to understand the association of spectral non-linearity in the thermal infrared (TIR) region. Using model-based unmixing [2], the non-linear mixing model (NLMM) can be formulated using a function $f$ as in eqn. (1),

$$
r_{i}=f\left(m_{l}, \xi_{l}, \rho, \eta ; \lambda\right)
$$

where, $r \in \Re^{n \times 1}$ and $m_{l} \in L_{n \times l}($ library $) ; l=1, \ldots, q$. The abundance vector $\xi_{l}=\xi_{1}, \xi_{2}, \ldots, \xi_{q}^{T}$. Such unknown nonlinear function depends upon the spatial extent, material fractions, media parameters $(\rho)$ and noise levels $(\eta)$. The physical models do not easily lend themselves to simple mathematical solutions. The main difficulty is how to model these nonlinearities when much of the necessary information is not available. Hence, tackling and addressing the spectral nonlinearities is the most difficult task. However, by using the Planck radiation law, the emissivity interaction of intimate mixtures in thermal infrared domain could be define accurately [3]. Most of the thermal hyperspectral remote sensing researchers $[4,5]$ believed that spectral mixing phenomenon in the emissive (TIR) domain is purely a linear process. Taking a clue from the parameters contributing to non-linearity [2], the uncertainties in unmixing of TIR hyperspectral image cube are investigated in this paper.

\section{Methodology}

A schematic flow chart of the proposed methodology is shown in Fig. 1. The steps will be further explored in the following sections to show the existence and cause of spectral non-linearity in the emissive domain.

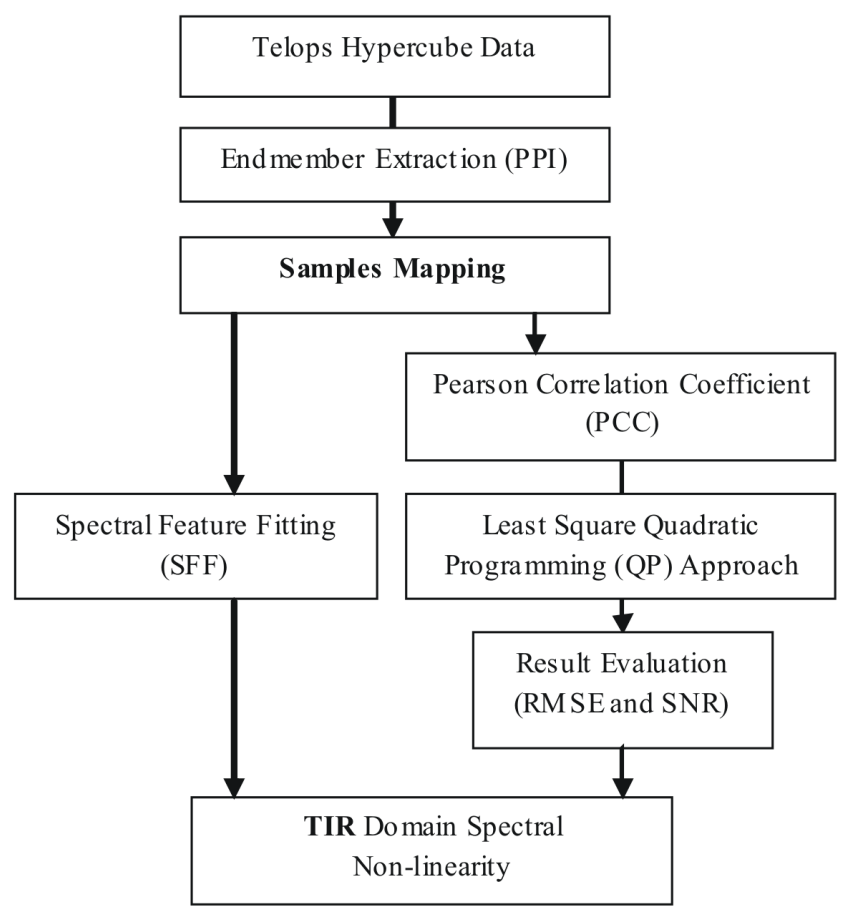

Figure 1. Hierarchical diagram depicting the adopted methodology. 


\subsection{Thermal Hyperspectral Data Acquisition}

In this study, an infrared imaging instrument (Telops Hyper-Cam-LW) available at Gabriel Lippmann Institute, Luxembourg is used to collect hyperspectral data in laboratory conditions which covers a spectral range of 8 to $12 \mu \mathrm{m}$. Three geological samples- limestone, alunite and quartz are used in this research. For emissivity retrieval, the samples are arranged as shown in Fig. 2.

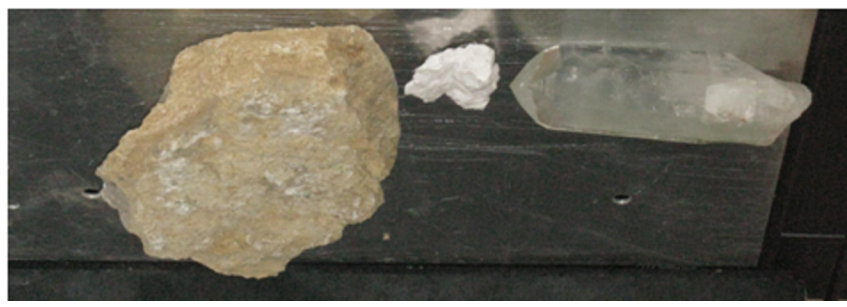

Figure 2. Diagram showing surface of limestone, alunite and quartz sample respectively.

The Telops Hyper-Cam acquired a complete TIR hyperspectral data $(128 \times 128 \times 81)$ with an instantaneous field-ofview of $0.35 \mathrm{mrad}$. It forms a hyperspectral image cube with $\mathrm{n}=81$ spectral bands of number of pixels, $\mathrm{p}=16384$ as shown in Fig. 3.

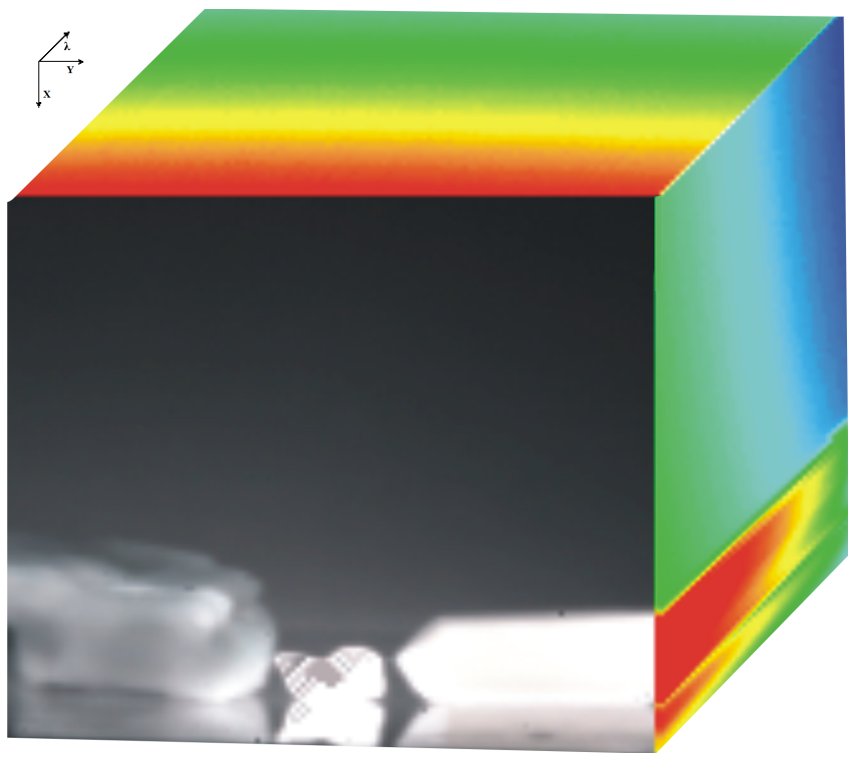

Figure 3. Telops Hyper-Cam measured TIR hyperspectral image cube (Image cube courtesy: Gabriel Lippmann Institute, Luxembourg).

\subsection{Endmember Extraction}

In the ENVI software, the noise in the data is reduced using Minimum Noise Fraction (MNF) technique and then Pixel Purity Index (PPI) are used for automatic endmembers extraction. In the image, the spectrally pure pixels are computed by projecting $n$-dimensional scatter plots onto the selected random vector for the threshold factor of 2.5 and iterations of 10,000 . The nine endmembers showing characteristic absorption features (representing the chosen samples) are selected. Finally, these selected optimal set of endmembers are used for further analysis.

\subsection{Spectral Unmixing}

After the derived endmembers, the Pearson Correlation Coefficient (PCC) is used to match these endmembers to each pixel of the image scene by Linear Mixing Model (LMM) developed in $[6,7]$. Here, an iterative procedure based fully constrained quadratic programming algorithm is used to unmix each pixel of the hypercube [8]. It produces a vector of fractional abundances for individual endmember on pixel-bypixel basis and generates nine classified images representing each endmember.

\subsection{Accuracy Assessment}

The Root-Mean-Square Error (RMSE) and generalized Signal-to-Noise-Ratio (SNR) is used to measure the uncertainties between the original $\left(r_{i}\right)$ and the modeled $\left(M_{i}\right)$ image pixel spectra. The RMSE and SNR is defined as in eqn. (2),

$$
\begin{gathered}
R M S E=\sqrt{\frac{1}{n} \sum_{i=1}^{n} \eta(\lambda)_{i}^{2} ; \text { where }, \eta=\left\|r_{i}-M_{i}\right\|} \\
S N R=10 \times \log _{10}\left(\frac{\sum_{i=1}^{n}\left\|r_{i}-M_{i}\right\|^{2}}{\sum_{i=1}^{n}\|r\|^{2}}\right) d B
\end{gathered}
$$

Where, $n$ is the total number of wavelength $(\lambda)$ channels.

\section{Results and Discussions}

Using Spectral Feature Fitting (SFF) technique, the image is mapped for limestone, alunite and quartz respectively as shown in Fig. 4. In Fig. 4 (a) and (b), it is clearly seen that limestone and alunite boundaries are completely mapped. On the contrary, some portions ( $\sim 2-3$ inches) at the extreme left of quartz crystal (dark color pixels) remain unmapped (Fig. 4 (c)). Also in Fig. 4 (b), the extreme left of the quartz crystal (bright color pixels) are mapped as a mixture of alunite and quartz. Certain facets of quartz crystal showing the alunite signature is not expected, if the process has been linear and single scattering. Further, unmapped regions of limestone, alunite and quartz may be caused by interaction of emitted energy coming from the objects of different thermal properties and temperatures. Variation in thermal conductivity will determine the rate of the dissipation of heat, and hence, the brightness temperature of the target over time. For example, the thermal conductivity at room temperature $\left(20{ }^{\circ} \mathrm{C}\right)$ of quartz mineral $\left(20 \mathrm{~W} / \mathrm{m}^{\circ} \mathrm{C}\right)$ is relatively higher than alunite $\left(5 \mathrm{~W} / \mathrm{m}{ }^{\circ} \mathrm{C}\right)$ and limestone $\left(6 \mathrm{~W} / \mathrm{m}^{\circ} \mathrm{C}\right)$. Under these circumstances, objects heated at specified constant temperatures will reach thermal equilibrium with the ambiance at different 
rates. This temperature gradient will result in differential radiance from the objects and hence, bouncing of photons yield non-linear mixing. There could be the coupling of emitted radiation from different surface components of limestone, alunite and quartz crystal, which could result to such spectral non-linearity and uncertainty zone.

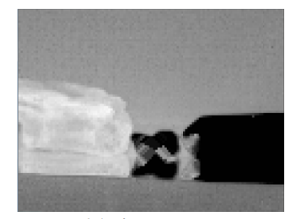

(a.) Limestone (b.) Alunite

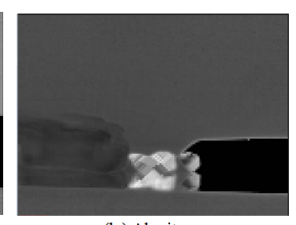

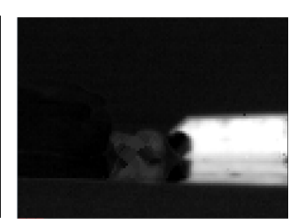

(c.) Quartz
Figure 4. Hypercube mapping with (a.) Limestone, (b.) Alunite, and (c.) Quartz using SFF.

The thermal-infrared radiance directly consists of reflected and emitted radiance from the adjacent scene samples. The apparent emissivity is reduced by reflected radiation because the emissivity $(\varepsilon)$ and the reflectivity $(\rho)$ is always complementary to each other (Kirchhoff's law). If materials are close enough, then the radiation emitted by each material is absorbed by the adjacent materials. Then it may be emitted later by the other materials. This process of absorption and emission give rise to higher non-linearity. In some cases, emitted radiation from one sample has been transmitted through the other adjacent sample and then emitted towards the sensor. Thus, the presence of alunite spectral feature on certain faces of quartz sample conveys that the emission spectroscopy is clearly not a case of linear spectral mixing. In Fig. 5 the elliptical portion precisely shows the higher uncertainty area, due to the dominance of alunite emissivity over quartz crystal.

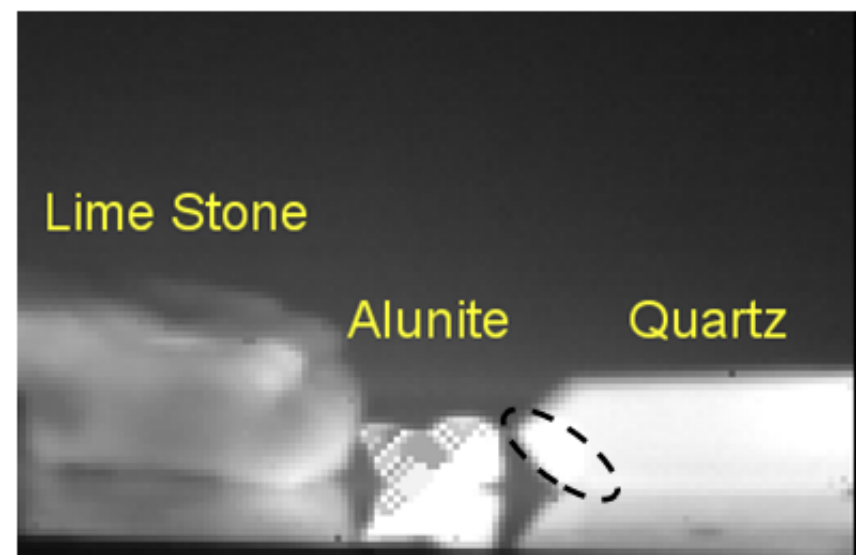

Figure 5. TIR hyperspectral data showing unmapped area (dotted ellipse) at extreme left of quartz crystal.

The derived emissivity spectra for limestone, alunite, quartz, and mixed alunite-quartz zone (most uncertain elliptical region) are shown in Fig. 6.

Fig. 7 shows the fractional abundance images for each endmember, obtained from the Discrete Wavelet Transformation (DWT) based linear spectral unmixing [6, 7]. In these

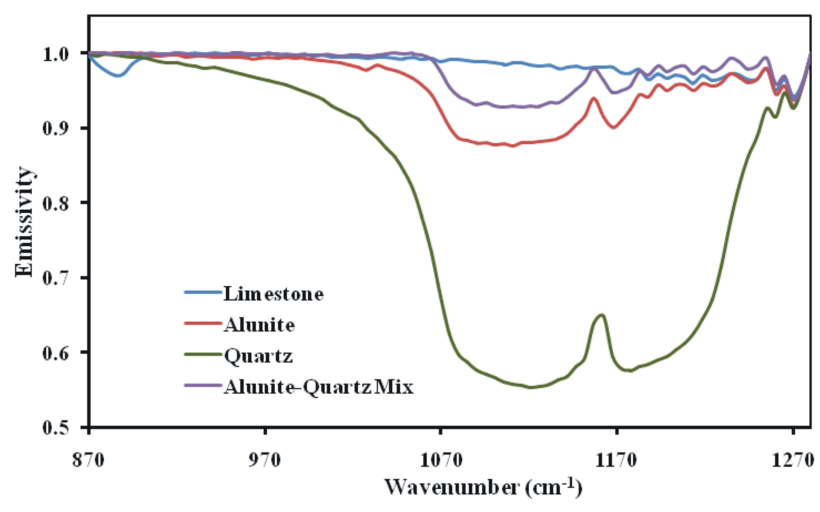

Figure 6. Derived emissivity profiles for limestone, alunite, quartz, and mixed alunite-quartz.

abundance maps, the dark black pixels represent the lowest fractions value $(0)$, different shades of grey color indicate increasing abundance, and exactly bright white pixels indicate the maximum fractions value (1). Thus, the endmember 1 to 4 are identified to represent quartz, 5 and 6 as limestone, 8 and 9 as alunite. The endmember 7 clearly indicates that it is a mixture of alunite and quartz. Pixels representing value 1 in the classified map typically indicate purest pixels. Whereas, pixels with values lower than 1 indicate mixed nature of spectra. The pixels that could not be resolved with the chosen set of endmembers usually appear dark (0 values).
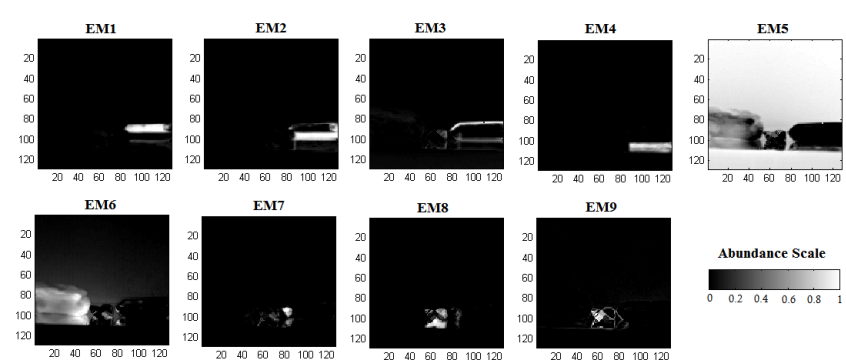

Figure 7. Fractional abundance maps of extracted nine endmembers (EM).

To understand the variations in spectral similarity between pure pixels and mix pixels, a RMSE image (Fig. 8) is obtained after taking root mean square difference between actual and estimated modeled spectra for each image pixel. The elliptical area in Fig. 8 can be qualitatively compared with Fig. 5 for maximum RMSE zones (yellow color dotted ellipse). This can be addressed more clearly by plotting image pixel's SNR peaks in a 3D shaded surface (Fig. 9). The encircled portion in Fig. 9 indicates minimum SNR peaks corresponding to maximum uncertainty.

The analysis of SFF mapped images, RMSE and SNR image clearly indicates the regions of spectral mixing. These pixels show abnormal deviations from the reality, and certainly are the result of radiation mixing/contamination from adjacent objects. This is mainly attributed to multiple scattering from nearby objects. The magnitude of the multiple scattering depends on the relative fractional covers of the samples and the wavelength. 


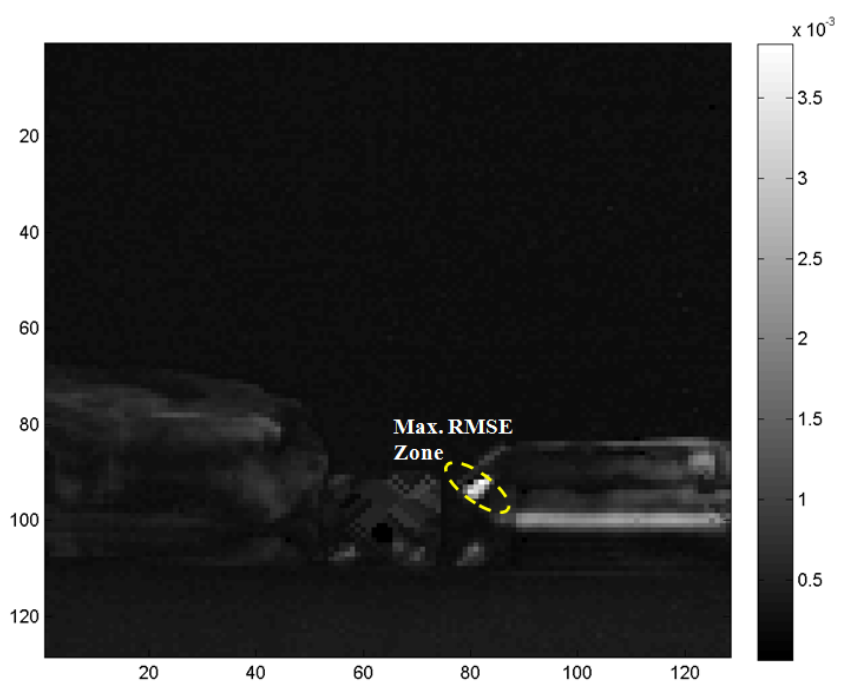

Figure 8. RMSE image created using pure signatures of quartz, alunite, and limestone.

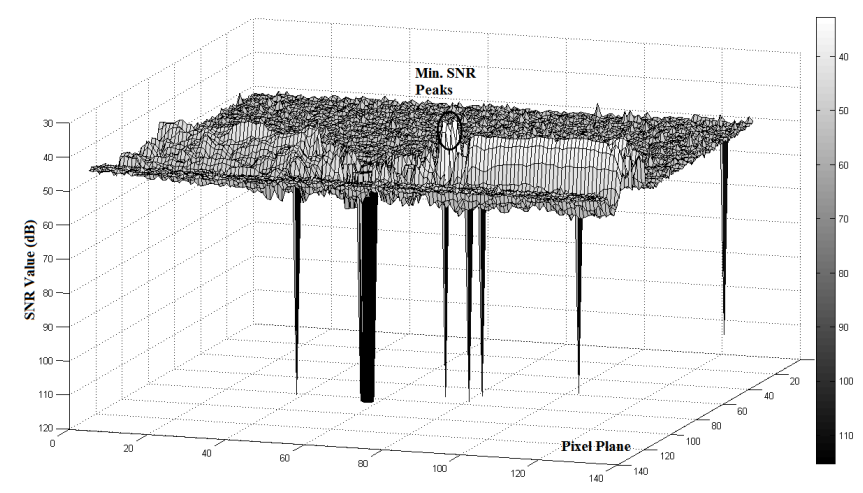

Figure 9. 3D view of signal-to-noise-ratio of each image pixel.

\section{Conclusions}

It can be concluded that in thermal infrared region, the photons undergo multiple scattering which is the result of non-linear mixing. It is generally caused by coupling of emitted radiance between spatially adjacent objects with heterogeneous thermal properties. In particular, spatial variations on thermal properties could be used during intimate mixtures mapping to formulate the non-linear function. The analytical procedure presented in this work can be used as a guideline for evaluating the mapping accuracy of hyperspectral images in the TIR region.

\section{Acknowledgements}

The authors are thankful to Prof. Dr. Schlerfet at Gabriel Lippmann Institute, Luxembourg and Telops, Canada for ac- quiring hypercube. Author acknowledge Prof. D. Ramakrishnan at Indian Institute of Technology Bombay, India for the project grant support.

\section{REFERENCES}

[1] M. Schlerf, G. Rock, P. Lagueux, F. Ronellenfitsch, M. Gerhards, L. Hoffmann, and T. Udelhoven, "A Hyperspectral Thermal Infrared Imaging Instrument for Natural Resources Applications," MDPI-Remote Sensing, vol. 4, pp. 3995-4009, 2012.

[2] K. D. Singh and D. Ramakrishnan, "Uncertainties in Unmixing of Multi-phase Hyperspectral Data in Reflective Region: Effects of Texture and Fabric," Journal of Hyperspectral Remote Sensing, vol. 5, no. 3, pp. 086-100, 2015.

[3] G. Fontanilles and X. Briottet, "A nonlinear unmixing method in the infrared domain," Applied Optics, vol. 50, pp. 3666-3677, 2011.

[4] M. S. Ramsey and P. R. Christensen, "Mineral abundance determination: Quantitative deconvolution of thermal emission spectra," Journal of Geophysical Research, vol. 103, pp. 577-596, 1998.

[5] S. A. Nowicki and P. R. Christensen, "Rock abundance on Mars from the Thermal Emission Spectrometer," Journal of Geophysical Research, vol. 112, no. E05007, pp. 1-20, 2007.

[6] K. D. Singh, D. Ramakrishnan, and L. Mansinha, "Relevance of transformation techniques in rapid endmember identification and spectral unmixing: A hypespectral remote sensing perspective," in IEEE Geoscience and Remote Sensing Symposium (IGARSS), Munich, Germany, July 2012, pp. 4066-4069.

[7] K. D. Singh and D. Ramakrishnan, "A comparative study of signal transformation techniques in automated spectral unmixing of infrared spectra for remote sensing applications," International Journal of Remote Sensing, vol. 38, no. 5, pp. 1235-1257, 2017.

[8] D. Heinz and C.-I. Chang, "Fully constrained least squares linear spectral mixture analysis method for material quantification in hyperspectral imagery," IEEE Trans. Geosci. Remote Sensing, vol. 39, no. 3, pp. 529545, 2001. 